\title{
Expression of E-cadherin in oral lichen planus
}

\author{
YONG DU and HAOBO LI
}

Department of Oral Medicine, The Second Hospital of Hebei Medical University, Shijiazhuang, Hebei 050000, P.R. China

Received October 20, 2014; Accepted July 9, 2015

DOI: $10.3892 /$ etm.2015.2654

\begin{abstract}
Oral lichen planus (OLP) is a common lesion of the oral mucosa that can progress to cancer. E-cadherin is involved in intercellular adherence and the pathogenesis, development and metastasis of tumors, and is considered to be an important indicator of tumor progression and prognosis. The aim of this study was to investigate the expression of E-cadherin in OLP in order to elucidate its role in the pathogenesis and malignant transformation of OLP and provide evidence to support the early diagnosis and treatment of OLP with malignant potential. OLP specimens $(n=52)$ and samples of normal oral mucosa $(n=41)$ as the control were collected. Immunohistochemical staining was conducted to reveal the expression of E-cadherin in the OLP samples and normal oral mucosa. It was observed that 25 of the 52 OLP specimens exhibited normal positive expression of E-cadherin and 27 exhibited abnormal positive expression, corresponding to an abnormal positive rate of $51.92 \%$. In the normal oral mucosa group, 39 of the 41 cases exhibited normal positive expression and 2 exhibited abnormal positive expression. The abnormal positive rate in the normal oral mucosa was $4.88 \%$, which was significantly lower than that in the OLP group. The significantly elevated rate of abnormal positive expression of E-cadherin in the OLP group indicates the involvement of E-cadherin in the malignant transformation of OLP and supports the malignant potential of OLP.
\end{abstract}

\section{Introduction}

Oral lichen planus (OLP) is a common lesion of the oral mucosa. Its specific pathogenesis is unknown, although it is considered to involve antigen-specific and antigen-nonspecific mechanisms. The antigen-specific mechanism involves antigen presentation by basal keratinocytes and antigen-specific keratinocyte killing by CD8 cytotoxic cells. In the non-specific mechanism, mast cell degranulation and matrix metalloproteinase activation are involved. It has been reported that OLP

Correspondence to: Dr Haobo Li, Department of Oral Medicine, The Second Hospital of Hebei Medical University, 215 Heping West Road, Shijiazhuang, Hebei 050000, P.R. China

E-mail: haobolicn@163.com

Key words: oral lichen planus, E-cadherin, immunohistochemical method shows cancerous characteristics with a malignant transformation rate of $0.4-6.5 \%$ (1-3). Due to the abnormal expression of certain oncogenes or anti-oncogenes in OLP, which serves as an indication of its risk of progression to cancer, the World Health Organization (WHO) has classified OLP as 'potentially malignant lesions' $(1,4)$. It has been demonstrated that the carcinogenesis of OLP involves a combination of factors, which induce disorders of epithelial cell proliferation and apoptosis, and abnormalities of oncogenes, anti-oncogenes or certain signaling pathways, resulting in the malignant development of OLP (5-7). In recent years, the malignant potential of OLP has been controversial. Certain researchers consider that OLP does not show any higher malignancy rate than other types of benign damage of the oral mucosa, and that the epithelial tissue of OLP is similar to that of benign lesions but different from potentially malignant or cancerous oral tissues (8). Three tumor suppressor gene loci of OLP were found to be significantly different from those of low-grade dysplasia, high-grade dysplasia or oral squamous cell carcinoma (OSCC), but not different from benign fibroma (8). Thus, scientific assessment would be possible for the malignant risk of OLP if specific biomarkers were discovered.

E-cadherin, a type-I cadherin, is a transmembrane glycoprotein that mediates $\mathrm{Ca}^{2}$-dependent homotypic cell-cell adhesion. E-cadherin is mainly expressed in the epithelial cells of humans and animals, and is the first cadherin expressed during mammalian development. E-cadherin is essential for cell differentiation during embryonic development, and is involved in tissue and organ development. It is vital to the maintenance of the polarity of cells and cohesion of tissues, as well as to the morphology of epithelial cell and tissue integrity. Therefore, the E-cadherin-mediated junctions between cells play an important role in tumorigenesis, since impairment of the junction will result in increased cellular invasion and migration, facilitating the growth and proliferation of tumor cells at metastatic positions to form new lesions (9). Studies have shown that E-cadherin mediates the non-adherent growth of a variety of cells $(10,11)$. There is also evidence that cleavage fragments of E-cadherin are involved in cellular junction destruction, cell migration, invasion and abnormal signal activation, which indicates a promoting role of E-cadherin in cancer progression. Studies have found the abnormal expression of E-cadherin in a variety of malignant tumors of epithelial origin, such as colorectal carcinoma, gastric carcinoma, esophageal carcinoma, hepatoma, lung neoplasms, breast carcinoma, prostate cancer and endometrial malignant tumors (12-14). This reveals that E-cadherin 
might have a close association with the pathological features and biological behavior of tumors. E-cadherin not only plays an important role in cancer pathogenesis and metastasis, but is also greatly involved in the development of precancerous lesions, indicated by the fact that the abnormal expression of E-cadherin has been observed in oral leukoplakia and cervical intraepithelial neoplasia $(15,16)$. Therefore, it is suggested that E-cadherin might be a suitable indicator for the early detection, diagnosis and prevention of tumors.

The clinical evaluation of cancerous tissue is primarily based on the histological appearance of epithelial dysplasia in pathological sections at present. In recent years, the application of biomarkers has contributed to objective evaluation of the stage of OLP, and to the prediction of the potential risk of malignant transformation (17). Thus, the improvement of the specificity and reliability of biomarkers remains the core task in this research field. The discovery of a reliable biomarker that indicates the transformation from OLP to OSCC will greatly improve the accurate and scientific assessment of OLP. E-cadherin expression has been reported in OSCC (18), but few studies have been published concerning its expression in OLP. Therefore, whether E-cadherin participates in cancerous changes to OLP remains to be investigated.

The aim of the present study was to demonstrate the expression of E-cadherin in OLP by immunochemistry, and to elucidate the potential role of E-cadherin in the pathogenesis of OLP. This may provide theoretical evidence to aid the early diagnosis and clinical treatment of OLP.

\section{Materials and methods}

Patients and samples. In total, 52 OLP specimens were obtained from patients admitted to the Second Hospital of Hebei Medical University (Shijiazhuang, China) between 2005 to 2012. This study was approved by the ethics committee of the Second Hospital of Hebei Medical University (Shijiazhuang, China). Written informed consent was obtained from the patients or their families. None of the patients had received any treatment prior to sampling. Following fixation in $10 \%$ formalin, the tissue specimens were embedded in paraffin, stained with hematoxylin and eosin (H\&E) and analyzed by a pathologist to obtain a confirmed diagnosis. The samples were obtained from a study group of 28 males and 24 females, with ages ranging from 38 to 73 years (mean age, 52.6 years). There were 41 normal specimens in the control group, comprising specimens of oral mucosa tissue harvested from alveolar bone cyst paraneoplastic plastic surgery that were processed and analyzed by pathology. There were 22 males and 19 females in the control group with age from 21 to 68 years (mean, 31.6 years).

Reagents. Rabbit anti-human monoclonal E-cadherin antibody (BA0474) and biotin-labeled goat anti-rabbit IgG antibody (BA1003) were acquired from Wuhan Boster Biological Technology, Ltd. (Wuhan, China). The streptavidin peroxidase (SP) immunohistology kit and diaminobenzidine (DAB) staining kit were obtained from Hebei Bio-High Technology Development Co., Ltd. (Shijiazhuang, China).

Immunohistochemistry. All specimens were fixed in $10 \%$ formaldehyde, embedded in paraffin, cut into three consecutive slices $5 \mu \mathrm{m}$ in thickness, and respectively subjected to $\mathrm{H} \& \mathrm{E}$ staining or E-cadherin immunohistochemistry, or served as a negative control.

Immunohistochemical SP staining was conducted according to a published procedure (19), with rabbit anti-human monoclonal E-cadherin antibody as primary antibody and biotin-labeled goat anti-rabbit IgG as secondary antibody. The samples of normal oral mucosa, known to be E-cadherin positive, were taken as the positive control. A negative control was established using phosphate-buffered saline (PBS) instead of the primary antibody.

The immunohistochemical analysis was conducted as follows: Sections were dewaxed in xylene and rehydrated through graded concentrations of ethanol. The sections were then incubated in 3\% hydrogen peroxide in methanol for $25 \mathrm{~min}$ to block endogenous peroxidase. Antigen retrieval was performed in a microwave at $92-98^{\circ} \mathrm{C}$ for $15 \mathrm{~min}$. Sections were incubated with rabbit anti-human monoclonal E-cadherin antibody (1:200) at $37^{\circ} \mathrm{C}$ for $30 \mathrm{~min}$, followed by washing with PBS for three times. Sections were incubated with biotinylated goat anti-rabbit secondary antibodies (1:300) for $25 \mathrm{~min}$ at $37^{\circ} \mathrm{C}$ and then incubated with peroxidase-conjugated streptavidin for $20 \mathrm{~min}$ at $37^{\circ} \mathrm{C}$.

The peroxidase reaction was developed in PBS using hydrogen peroxide as substrate and DAB as a chromogen. Sections were counterstained with hematoxylin, dehydrated in graded alcohol, and evaluated under a light microscope.

The staining distribution and intensity of E-cadherin were observed following immunohistological staining. The normal positive expression of E-cadherin was defined as the presence of brownish or yellow staining of the cellular membrane, while abnormal positive expression was defined as the presence of lines or dots of yellow or brownish granules in the cytoplasm with or without the same deposits on the cell membrane. The absence of staining was rated as negative.

Statistical analysis. Data were analyzed using SPSS software, version 17.0 (SPSS, Inc., Chicago, IL, USA). The differences were analyzed by $\chi^{2}$ test, with $\mathrm{P}<0.05$ considered to indicate a statistically significant difference.

\section{Results}

Expression of E-cadherin in normal oral mucosa and OLP. Among the 41 specimens of normal oral mucosa, 39 showed normal positive expression and 2 exhibited abnormal positive expression of E-cadherin, corresponding to an abnormal positive rate of $4.88 \%$. In the 52 OLP specimens, 25 presented normal positive expression and 27 showed abnormal positive expression of E-cadherin, corresponding to an abnormal positive rate of $51.92 \%$. There was a significant difference in abnormal positive rates between the two groups $\left(\chi^{2}, 23.64\right.$; $\mathrm{P}<0.05$; Table I; Figs. 1 and 2).

Association between the expression of E-cadherin and clinicopathological parameters. No statistically significant difference was identified between patients in the OLP group grouped according to gender or age $<50$ or $\geq 50$ years with regard to the abnormal expression of E-cadherin $(\mathrm{P}>0.05)$, as shown in Table II. 
Table I. Expression of E-cadherin in the normal mucosa and oral lichen planus groups.

\begin{tabular}{lccccc}
\hline Tissue type & $\mathrm{n}$ & Normal positive & Abnormal positive & Abnormal positive rate, $\%$ & $\chi^{2}$ \\
\hline Normal mucosa & 41 & 39 & 2 & 4.88 & 23.64 \\
Oral lichen planus & 52 & 25 & 27 & 51.92 & $<0.05$ \\
\hline
\end{tabular}

Table II. Association between E-cadherin expression and clinical characteristics in all patients with oral lichen planus.

\begin{tabular}{|c|c|c|c|c|c|c|}
\hline Variable & $\mathrm{n}$ & Normal positive & Abnormal positive & Abnormal positive rate, $\%$ & $\chi^{2}$ & P-value \\
\hline Gender & & & & & 0.089 & $>0.05$ \\
\hline Male & 28 & 14 & 14 & 50.00 & & \\
\hline Female & 24 & 11 & 13 & 54.17 & & \\
\hline Age, years & & & & & 0.930 & $>0.05$ \\
\hline$<50$ & 18 & 7 & 11 & 61.11 & & \\
\hline$\geq 50$ & 34 & 18 & 16 & 47.06 & & \\
\hline
\end{tabular}

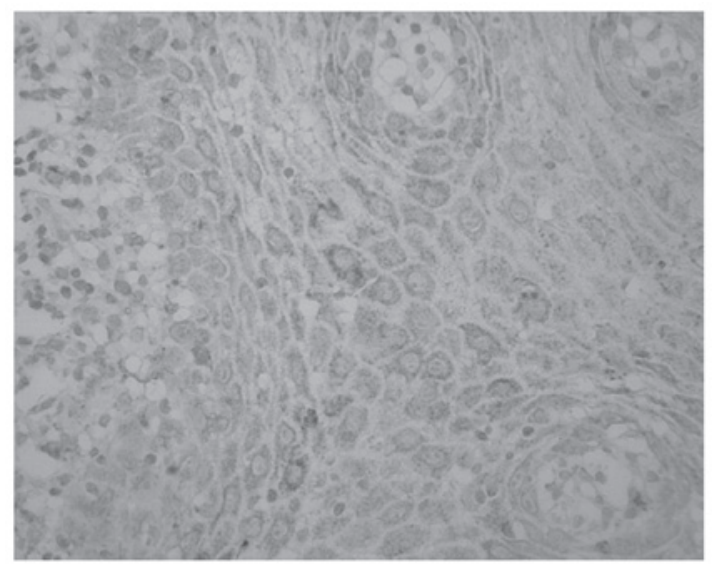

Figure 1. Expression of E-cadherin in normal tisssues. Streptavidin peroxidase immunohistochemistry (magnification, $\mathrm{x} 400$ ).

\section{Discussion}

Oral lichen planus (OLP) is an autoimmune inflammatory disease of the oral mucosa. The pathogenesis of OLP is associated with chronic focal injury, drug stimulation, mental stress, systemic diseases, and bacterial and viral infection (20). OLP is a chronic disorder of oral mucosal basal keratinocytes that is associated with a comprised immune system, caused by exogenous or autologous antigens and mediated by a cellular immune response. Chronic OLP lesions are thought to be precancerous with the potential to become malignant. OLP may transform into oral squamous cell carcinoma (OSCC) via a series of complex processes. Carcinogenesis results from a comprehensive network of components, and involves many factors such as local immunodepression, viral infection, carcinogens and cytokines. Oncogene activation, chromosomal mutation and anti-oncogene abnormalities are required to occur before malignant transformation eventually occurs. A previous study concerning the mechanisms by which OLP becomes cancerous and the associated genes has drawn wide-

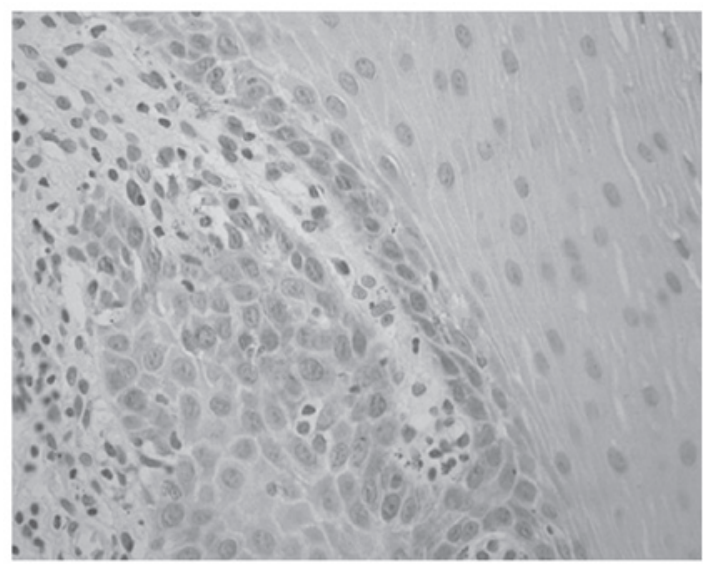

Figure 2. Expression of E-cadherin in oral lichen planus tisssues. Streptavidin peroxidase immunohistochemistry (magnification, $\mathrm{x} 400$ ).

spread attention (21). Clarification of the mechanism would be of great importance to the early prevention, diagnosis and treatment of OLP with malignant potential.

E-cadherin, a calcium-dependent intercellular adhesion molecule, is a member of the cadherin superfamily. Its coding gene is located on chromosome 16q22.1, with its $\mathrm{N}$ terminus located outside the cell while its $\mathrm{C}$ terminus is inside. E-cadherin is an important cell adhesion molecule, which is involved in the regulation of intercellular and cell-matrix adhesion, and the maintenance of tissue morphology and integrity. E-cadherin-mediated adhesion plays an important role in the maintenance of the polarity of epithelial cells and the inhibition of cell migration $(22,23)$. As a vital tumor suppressor molecule, E-cadherin functions primarily by enhancement of intercellular adhesion and by inhibition of cellular proliferation. The abnormal gene expression of E-cadherin is closely associated with the occurrence, development and metastasis of tumors, and is strongly associated with the degree of tumor differentiation, lymph node metastasis, distant metastasis and recurrence rate, 
suggesting that the evaluation of abnormal E-cadherin gene expression may be a vital indicator of tumor progression and prognosis $(24,25)$. Studies on the expression of E-cadherin in a variety of tumors have observed the presence of E-cadherin gene mutations and subsequent functional changes, in the form of reductions or loss of E-cadherin expression accompanied by cancer invasion and increased metastasis (26-28). This indicates that the reduction or loss of E-cadherin may be a notable change associated with epithelial-mesenchymal transition. Extracellular signals activate various transcription factors through diverse signal transduction regulatory mechanisms, and these can then regulate the expression of downstream proteins. As the core of this regulatory network, E-cadherin plays an important role in the progression, invasion and metastasis of tumors by endowing migrated tumor cells with an epithelioid phenotype that results in further proliferation to form a metastatic lesion (29-31). The present study is among the first to report on whether E-cadherin plays a role in the occurrence, development and progression of OSCC from OLP.

In the present study, the expression of E-cadherin was detected in 41 normal oral mucosa specimens and 52 OLP specimens. The results showed a significant difference between the two groups, with significantly abnormal expression in the OLP group. The loss of membranous expression of E-cadherin is involved in the malignant transformation of OLP. Therefore, we consider that OLP to be potentially malignant lesion. Oral leukoplakia is a common precancerous lesion of the oral mucosa, as is OLP. E-cadherin exhibits abnormal expression in oral leukoplakia (32), which is comparable with the abnormal expression in OLP observed in the present study. This supports the association of E-cadherin with cancerous potential.

In summary, the abnormal positive expression of E-cadherin is significantly elevated in OLP patients, which supports the malignant potential of OLP. The results of the present study may help to clarify the canceration processes underlying OLP. However, whether E-cadherin may be used as a determinate observation index of OLP canceration required verification in future studies.

\section{References}

1. Georgakopoulou EA, Achtari MD, Achtaris M, Foukas PG and Kotsinas A: Oral lichen planus as a preneoplastic inflammatory model. J Biomed Biotechnol 2012: 759626, 2012

2. Bombeccari GP, Guzzi G, Tettamanti M, Gianni AB, Baj A, Pallotti F and Spadari F: Oral lichen planus and malignant transformation: A longitudinal cohort study. Oral Surg Oral Med Oral Pathol Oral Radiol Endod 112: 328-334, 2011.

3. Shen ZY, Liu W, Feng JQ, Zhou HW and Zhou ZT: Squamous cell carcinoma development in previously diagnosed oral lichen planus: De novo or transformation? Oral Surg Oral Med Oral Pathol Oral Radiol Endod 112: 592-596, 2011.

4. Warnakulasuriya S, Johnson NW and Van der Waal I: Nomenclature and classification of potentially malignant disorders of the oral mucosa. J Oral Pathol Med 36: 575-580, 2007.

5. Van der Waal I: Potentially malignant disorders of the oral and oropharyngeal mucosa; terminology, classification and present concepts of management. Oral Oncol 45: 317-323, 2009.

6. Safadi RA, Al Jaber SZ, Hammad HM and Hamasha AA: Oral lichen planus shows higher expressions of tumor suppressor gene products of p53 and p21 compared to oral mucositis. An immunohistochemical study. Arch Oral Biol 55: 454-461, 2010.
7. Georgakopoulou EA, Troupis TG, Troupis G and Gorgoulis VG: Update of the cancer-associated molecular mechanisms in oral lichen planus, a disease with possible premalignant nature. J BUON 16: 613-616, 2011.

8. Accurso BT, Warner BM, Knobloch TJ, Weghorst CM, Shumway BS, Allen CM and Kalmar JR: Allelic imbalance in oral lichen planus and assessment of its classification as a premalignant condition. Oral Surg Oral Med Oral Pathol Oral Radiol Endod 112: 359-366, 2011.

9. Andrews JL, Kim AC and Hens JR: The role and function of cadherins in the mammary gland. Breast Cancer Res 14: 203, 2012.

10. Rodriguez FJ, Lewis-Tuffin LJ and Anastasiadis PZ: E-cadherin's dark side: Possible role in tumor progression. Biochim Biophys Acta 1826: 23-31, 2012.

11. Kang HG, Jenabi JM, Zhang J, Keshelava N, Shimada H, May WA, Ng T, Reynolds CP, Triche TJ and Sorensen PH: E-cadherin cell-cell adhesion in Ewing tumor cells mediates suppression of anoikis through activation of the ErbB4 tyrosine kinase. Cancer Res 67: 3094-3105, 2007.

12. Tsalikidis C, Papachristou F, Pitiakoudis M, Asimakopoulos B, Trypsianis G, Bolanaki E, Syrigos KN and Simopoulos C: Soluble E-cadherin as a diagnostic and prognostic marker in gastric carcinoma. Folia Med (Plovdiv) 55: 26-32, 2013.

13. Wójcik-Krowiranda K, Forma E, Zaczek A, Bryś M, Anna MK and Bieńkiewicz A: Expression of E-cadherin and beta1-integrin mRNA in endometrial cancer. Ginekol Pol 84: 910-914, 2013 (In Polish).

14. Hashiguchi M, Ueno S, Sakoda M, Iino S, Hiwatashi K, Minami K, Ando K, Mataki Y, Maemura K, Shinchi H, et al: Clinical implication of ZEB-1 and E-cadherin expression in hepatocellular carcinoma (HCC). BMC Cancer 13: 572, 2013.

15. Auvinen E, Carpen O, Korpela T, Ronty M, Vaheri A and Tarkkanen J: Altered expression of ezrin, E-cadherin and $\beta$-catenin in cervical neoplasia. Neoplasma 60: 56-61, 2013.

16. Kyrodimou M, Andreadis D, Drougou A, Amanatiadou EP, Angelis L, Barbatis C, Epivatianos A and Vizirianakis IS: Desmoglein-3/ $\gamma$-catenin and E-cadherin $/ \beta$-catenin differential expression in oral leukoplakia and squamous cell carcinoma. Clin Oral Investig 18: 199-210, 2014.

17. Poomsawat S, Buajeeb W, Khovidhunkit SO and Punyasingh J: Overexpression of cdk4 and p16 in oral lichen planus supports the concept of premalignancy. J Oral Pathol Med 40: 294-299, 2011.

18. Supic G, Kozomara R, Jovic N, Zeljic K and Magic Z: Prognostic significance of tumor-related genes hypermethylation detected in cancer-free surgical margins of oral squamous cell carcinomas. Oral Oncol 47: 702-708, 2011.

19. Soslow RA, Dannenberg AJ, Rush D, Woerner BM, Khan KN, Masferrer J and Koki AT: Cox-2 is expressed in human pulmonary, colonic and mammary tumors. Cancer 89: 2637-2645, 2000.

20. Regezi JA, Sciubba JJ and Jordan RCK (eds): Oral Pathology: Clinical Pathologic Correlations. 6th edition. Elsevier Saunders, St. Louis, MO, 2012.

21. Sun L, Feng J, Ma L, Liu W and Zhou Z: CD133 expression in oral lichen planus correlated with the risk for progression to oral squamous cell carcinoma. Ann Diagn Pathol 17: 486-489, 2013.

22. Sleeman JP and Thiery JP: SnapShot: The epithelial-mesenchymal transition. Cell 145: 162. e1, 2011.

23. Wells A, Chao YL, Grahovac J, Wu Q and Lauffenburger DA: Epithelial and mesenchymal phenotypic switchings modulate cell motility in metastasis. Front Biosci (Landmark Ed) 16: 815-837, 2011.

24. Kolesnik AP, Shevchenko AI, Tumanskiǔ VA and Evseev AV: Effect of the intercellular adhesion molecule E-cadherin on the prognosis of non-small cell lung cancer. Arkh Patol 75: 30-33, 2013 (In Russian).

25. Horne HN, Sherman ME, Garcia-Closas M, Pharoah PD, Blows FM, Yang XR, Hewitt SM, Conway CM, Lissowska J, Brinton LA, et al: Breast cancer susceptibility risk associations and heterogeneity by E-cadherin tumor tissue expression. Breast Cancer Res Treat 143: 181-187, 2014.

26. Sekar P, Bharti JN, Nigam JS, Sharma A and Soni PB: Evaluation of p53, HoxD10 and E-cadherin status in breast cancer and correlation with histological grade and other prognostic factors. J Oncol 2014: 702527, 2014.

27. Deep G, Jain AK, Ramteke A, Ting H, Vijendra KC, Gangar SC, Agarwal C and Agarwal R: SNAI1 is critical for the aggressiveness of prostate cancer cells with low E-cadherin. Mol Cancer 13: 37, 2014. 
28. Nakagawa H, Hikiba Y, Hirata Y, Font-Burgada J, Sakamoto K, Hayakawa Y, Taniguchi K, Umemura A, Kinoshita H, Sakitani K, et al: Loss of liver E-cadherin induces sclerosing cholangitis and promotes carcinogenesis. Proc Natl Acad Sci USA 111: 1090-1095, 2014.

29. Voulgari A and Pintzas A: Epithelial-mesenchymal transition in cancer metastasis: Mechanisms, markers and strategies to overcome drug resistance in the clinic. Biochim Biophys Acta 1796: 75-90, 2009.
30. Chaffer CL and Weinberg RA: A perspective on cancer cell metastasis. Science 331: 1559-1564, 2011.

31. Kalluri R: EMT: When epithelial cells decide to become mesenchymal-like cells. J Clin Investig 119: 1417-1419, 2009.

32. von Zeidler SV, de Souza Botelho T, Mendonça EF and Batista AC: E-cadherin as a potential biomarker of malignant transformation in oral leukoplakia: A retrospective cohort study. BMC Cancer 14: 972, 2014. 